\section{A conversation with Jeffrey Settleman}

\section{By Kai-Jye Lou, Senior Writer}

Kinase inhibitors have had a major impact in cancer since Gleevec imatinib hit the market in 2001, but not all patients respond and nearly all responders eventually develop resistance. Although kinase inhibitor resistance has added complexity to the field, it has also driven researchers to uncover the underlying mechanisms and created opportunities for the development of next-generation drugs.

Gleevec is a small molecule BCR-ABL tyrosine kinase inhibitor marketed by Novartis AG to treat chronic myelogenous leukemia (CML), acute lymphoblastic leukemia (ALL) and gastrointestinal stromal tumors (GISTs).

SciBX spoke with Jeffrey Settleman about the growing list of resistance mechanisms and what drug developers and the broader oncology research community can do to address them. Settleman is senior director of discovery oncology at Roche's Genentech Inc. unit.

Resistance has been a problem for kinase inhibitors since the start. Indeed, even before the FDA approved Gleevec, researchers were publishing reports of resistance to the drug in patients. ${ }^{1,2}$ Those

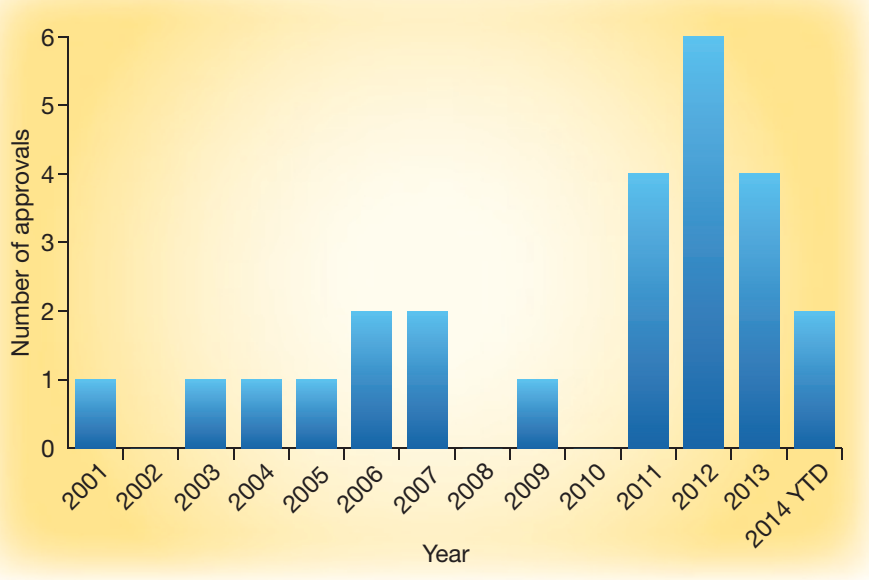

Figure 1. New kinase inhibitors approved in U.S. by year. Counts for 2014 exclude Chugai Pharmaceutical Co. Ltd.'s Alecensa alectinib, a small molecule anaplastic lymphoma kinase (ALK) inhibitor marketed in Japan to treat $\mathrm{ALK}^{+}$non-small cell lung cancer (NSCLC). The drug is not yet approved in the U.S.

Source: BioCentury archives; FDA studies and others helped guide the development of second-generation BCR-ABL inhibitors such as Sprycel dasatinib and Tasigna nilotinib, which entered the U.S. market in 2006 and 2007, respectively.

Sprycel is marketed by Bristol-Myers Squibb Co., and Tasigna is marketed by Novartis. Both are approved to treat patients with CML with resistance or intolerance to Gleevec or other therapies.

During the last three years, there has been a surge of approvals for kinase inhibitors in the U.S. Whereas 9 inhibitors reached the market between 2001 and 2009, 16 have been approved since the beginning of 2011 (see Figure 1, “New kinase inhibitors approved in U.S. by year”).

The new wave of drugs has been enabled by an improved understanding of cancer biology and the mechanisms underlying drug resistance. Even before joining Genentech in 2010, Settleman's focus had been on understanding these resistance mechanisms. His group has published multiple recent studies describing mechanisms that drive resistance to kinase inhibitors and strategies to bypass them. ${ }^{3-8}$

The conversation with Settleman follows.

SciBX: What have been some of the most important kinase inhibitor resistance mechanisms identified, and what impact has their discovery had on the field?

Jeffrey Settleman: This has been an area of intense study for the last several years, and these efforts have not only led to the identification of specific resistance mechanisms but also have informed strategies to develop novel inhibitors that can overcome resistance.

One example would be in the context of inhibition of the ABL kinase in CML. A number of second-generation drugs can overcome the frequently observed molecular mechanism of resistance that involves mutations in the kinase itself.

That theme has been extended to other kinases, for example, EGFR [epidermal growth factor receptor] and ALK [anaplastic lymphoma kinase], where secondary mutations in the kinase domain are seen that confer resistance to the drug being used to target it. The molecular nature of those resistance mechanisms has helped inform strategies to develop subsequent drugs that can overcome them.

I think this is a trend we'll see going forward for other kinases, where we are also starting to see resistance mechanisms emerge. An example is Bruton's tyrosine kinase (BTK), where there have been recent data suggesting a similar problem of mutation-driven, drug-acquired resistance that affects the kinase itself.

SciBX: What are the most interesting types of resistance mechanisms for drug development?

JS: Well, I think resistance mechanisms that stem from an obvious molecular defect, such as the ones I just described, are associated with a potentially straightforward clinical path. If a newly discovered molecule can overcome that established mechanism of resistance, then you already know exactly which patients to treat in the clinic. The preclinical modeling should be predictive, and one would expect to see clinical activity in patients whose cancers carry the defined lesion. 


\section{"From a practical standpoint, one has to see enough cases of a particular mechanism of resistance to justify a drug discovery effort." - Jeffrey Settleman, Genentech Inc.}

There are also so-called bypass mechanisms, where resistance doesn't necessarily involve direct mutational activation. These include feedback mechanisms, cellular differentiation mechanisms and epigenetic mechanisms, where the biology is not as straightforward as mutational activation of the primary target.

I think these latter mechanisms are much more challenging, from both the drug discovery and the drug development perspective. The biology is more complicated, which makes it more difficult to determine exactly what to target. This complex biology also makes it more challenging to anticipate such mechanisms and to identify and stratify patients where such resistance mechanisms may be relevant.

SciBX: What data do you need to see to decide whether there is a therapeutic opportunity?

JS: From a practical standpoint, one has to see enough cases of a particular mechanism of resistance to justify a drug discovery effort.

We're starting to see so many reports of different mechanisms of resistance around the same class of targets or the same drug. When there are multiple resistance mechanisms at play, then it becomes challenging to know where to start. We will certainly want to evaluate the prevalence of each mechanism as well as druggability.

Even in cases where we think we understand a particular resistance mechanism, it doesn't necessarily yield a path for drug discovery because the mechanism could involve a protein that we don't yet know how to drug. Alternatively, if the mechanism involves an entire pathway, we may not yet know which protein in the pathway will be the best one to drug.

SciBX: What have been the major challenges in translating these findings into better treatments?

$J S$ : There are a number of significant challenges. One challenge is developing combination treatments that are safe and well tolerated. I think all of us in the field recognize that single-agent approaches will ultimately be associated with some form of acquired drug resistance and that combination treatments will be critically needed to manage drug resistance.

All of these kinase inhibitors have some degree of toxicity associated with their use, so as we work to develop combinations that could prevent or overcome resistance, there is going to be the potential for increased toxicity.

Combination treatments are also challenging in the sense that we can't always predict what the mechanism of resistance will be. For example, with the STAT3 [signal transducer and activator of transcription 3] feedback activation mechanism, we don't yet have a clear-cut way to identify the patients who will respond to a treatment in a manner that invokes this feedback mechanism.

Another challenge is to predict the relevant mechanisms at play in an individual patient. Most of the patients being treated with these targeted agents are in an advanced stage of disease where there are likely multiple lesions present. These may evolve differently under drug pressure, leading to multiple distinct resistance mechanisms within the same patient. This could then demand treatment with multiple different agents in combination to manage the various coexisting resistance mechanisms.

Finally, there is also heterogeneity within tumors, which we are increasingly recognizing these days. Even within an individual tumor lesion, there could be more than one escape mechanism.

SciBX: What would you like to see the oncology research community do more of to address resistance against kinase inhibitors?

JS: Actually, I think researchers are taking a pretty aggressive approach to understanding this problem, and I've been pleased to see that the field has really pushed hard on this question.

Clinically, I think everyone is interested in seeing more data from biopsies collected from patients progressing on therapy. But we're limited in our ability there because biopsies often require invasive procedures, and many patients simply don't wish to be re-biopsied. So, we are currently challenged somewhat in terms of the availability of information about disease progression in clinical settings.

There is far more data available describing what happens when we model resistance in cell culture, but that information may not be as predictive-or relevant-as what we are actually seeing in patients. Thus, I think gathering more information from clinical studies in the context of disease progression is ultimately going to be quite important.

\section{SciBX: Where should more effort be spent?}

JS: There are a few topics that I think would be quite interesting. One of the areas of interest in my laboratory has been in the epigenetic mechanisms of drug resistance and the nature of chromatin states that play a role in drug resistance.

I think the epigenetics field is really emerging in a substantial way in terms of understanding the molecular basis of chromatin changes, but there are more limited efforts in the area of epigenetics in the context of drug resistance in cancer cells.

I also think it would be useful to have a better understanding of what's going on in single cells when resistance develops. I mentioned heterogeneity within tumors, and I think that we will ultimately have to study that heterogeneity at the level of individual cells.

We are starting to see technologies emerge that can interrogate the genome and biochemical features at the single-
"I think all of us in the field recognize that single-agent approaches will ultimately be associated with some form of acquired drug resistance."

- Jeffrey Settleman, Genentech Inc. cell level, and I think advances in these areas could really be useful for understanding what's going on in a tumor cell population that determines the overall response to treatment.

SciBX: How do you think all these efforts to identify and circumvent kinase inhibitor resistance mechanisms will ultimately pan out in terms of patient care?

JS: I think it's really hard to imagine that any single-agent treatment will ever be truly effective because we know that there is a heterogeneous nature 


\section{ANALYSIS}

to tumors where there are multiple cellular phenotypes present-and these different cells seem to show distinct responses to single agents.

We will undoubtedly be enhancing our understanding of the nature of intrinsic and acquired resistance mechanisms in tumors and how tumor heterogeneity contributes. This will certainly help inform strategies that ultimately benefit patients and perhaps even bring about cures.

Combined with new drug discovery efforts to target challenging proteins that drive resistance, such as STAT3, and figuring out whether such agents could be safely combined to treat patients-this is what we are looking at going forward.

SciBX: Are there analogies or lessons from other non-oncology fields?

JS: When considering combination therapy, one thing that I often hear is, 'Do I think that this is going to be analogous to the HIV cocktail therapy approach', which has been very successful in managing that disease.

I would say that, in theory, a similar kind of cocktail approach could ultimately be useful in the cancer setting, but one of the major differences with HIV cocktail therapy is that we are targeting a foreign proteome, whereas with cancer we're essentially drugging ourselvesour normal tissues as well as the tumor.

\section{TRANSLATIONAL NOTES}

Thus, the ability to combine treatments in a safe manner really becomes a much more substantial challenge than I think it has been for the discovery and development of HIV cocktail therapies. The analogy can be extended to a certain point, and then we have to face different kinds of challenges in the cancer setting.

SciBX: Thank you for your time.

Lou, K.-J. SciBX 7(38); doi:10.1038/scibx.2014.1113

Published online Oct. 2, 2014

\section{REFERENCES}

1. Krystal, G.W. Drug Resist. Updat. 4, 16-21 (2001)

2. Weisberg, E. \& Griffin, J.D. Drug Resist. Updat. 4, 22-28 (2001)

3. Corcoran, R.B. et al. Oncotarget 2, 336-346 (2011)

4. Wilson, T.R. et al. Nature 487, 505-509 (2012)

5. Baas, T. SciBX 5(32); doi:10.1038/scibx.2012.832

6. Lee, H.-J. et al. Cancer Discov. 3,168-181 (2013)

7. Lee, H.-J. et al. Cancer Cell 26, 207-221 (2014)

8. Wilson, C. et al. Oncotarget 5, 7328-7341 (2014)

\section{COMPANIES AND INSTITUTIONS MENTIONED}

Bristol-Myers Squibb Co. (NYSE:BMY), New York, N.Y. Chugai Pharmaceutical Co. Ltd. (Tokyo:4519), Tokyo, Japan Food and Drug Administration, Silver Spring, Md.

Genentech Inc., South San Francisco, Calif. Novartis AG (NYSE:NVS;SIX:NOVN), Basel, Switzerland Roche (SIX:ROG; OTCQX:RHHBY), Basel, Switzerland 